\section{ADVANTAGES OF AN ANALYSIS OF SOIL-STRUCTURE INTERACTION IN A FREQUENCY DOMAIN}

\begin{abstract}
With the computer power available an analysis of a total soil-structure system has become possible. In this paper the advantages of an analysis performed in an frequency domain is shown: the condensation to the primary degrees of freedom, thereby conserving all the characteristic properties of the system, is straightforward; material damping based on rheological models can be considered easily; and even nonlinear problems may be investigated. The formulation is developed in a total displacement formulation, where the motion is driven by forces acting at the soil-structure interface, or more generally, at so-called interaction nodes. The total displacement formulation is compared with the classical approach, where the unknowns are the relative deformations with respect to the rigid base of the structure. These unknows are produced by inertial forces acting on the masses of the structure. In the conclusion the total displacement formulation in the frequency domain is suggested for structures with embedded elastic foundation built on a sub-grade which requires extended modeling for the mechanical representation of the structure and the sub-grade.
\end{abstract}

\section{ANALYSIS IN THE FREQUENCY DOMAIN}

Soil-structure interaction (SSI) problems can be investigated in a time domain or a frequency domain. As always, each approach has its own advantages and disadvantages. If nonlinearities have to be considered in the structure or the soil, the time domain cannot be avoided. Since the effect of wave propagation in infinite soil is well represented through the frequency-dependent dynamic stiffness of the sub-grade, the frequency domain is advantageous.

There are further advantages withan analysis in the frequency domain. One important feature is that the dynamic stiffness matrices can be condensed into selected primary degrees of freedom without changing the dynamic properties of the system. This allows the simulation of the dynamic behavior of the complete sub-grade with the impedance condensed to the soil-structure interface. Also, if the element-related stiffness matrices are derived from a Fouriertransformed wave equation, the exact dynamic stiffness matrices are obtained for the frequency considered.

As is well known, integral transformations can only be applied to linear systems. But in the literature procedures can be found where nonlinear problems are obtained in a time range of $0 \leq t$ $\leq T$ as the sum of the incremental results $\Delta \mathbf{u}_{i}(t), i=1,2, \ldots$ of the linear problems (Matthes,1982). The increments $\Delta \tilde{\mathbf{u}}_{i}(\omega)$ are calculated in the transformed space (the Laplace or Fourier space) and transformed back into the time domain, where the sum of the increments is checked for occurrences of nonlinearities. If not, the analysis is stopped; if yes, the necessary system changes are made and the next increment is calculated. 
The general "total displacement" formulation, which is familiar to us in the Finite Element Analysis, is used here in the theoretical development of SSI. More restricted formulations are derived from it.

\section{SOIL-SRUCTURE INTERACTION}

\subsection{Total Displacement Formulation}

The Finite Element Method (FEM) allows for the calculation of the deformation and the stresses in the structure due to - for each degree of freedom - aspecified load or specified displacement, respectively. In the case of specified displacements the displacement response contains possible rigid body motions. Thus the FEM analysis is always based on total displacements.

\subsubsection{Structure}

For a general structure, indicated in Fig.2.1, with the mass matrix $\mathbf{M}$, damping matrix $\mathbf{C}$ and stiffness matrix $\mathbf{K}$, the equation of motion and its Fourier transformation are

$\mathbf{M u ̈}+\mathbf{C u}+\mathbf{K u}=\mathbf{P}(t)$

$\tilde{\mathbf{K}}^{S}(\omega) \tilde{\mathbf{u}}^{S}(\omega)=\tilde{\mathbf{P}}^{S}(\omega)$

where in equation (2.2) the dynamic stiffness of the structure, identified with upper index $\mathrm{S}$, is given as

$\tilde{\mathbf{K}}^{S}(\omega)=\mathbf{K}-\omega^{2} \mathbf{M}+i \omega \mathbf{C}$

and $\tilde{\mathbf{u}}^{S}$ and $\tilde{\mathbf{P}}^{S}$ are the Fourrier- transformed nodal displacements and forces of the structure.

Alternatively, the dynamic stiffness matrix of a structure consisting of E elements can be established from dynamic elements, which are derived from the Fourier-transformed wave equation in the element

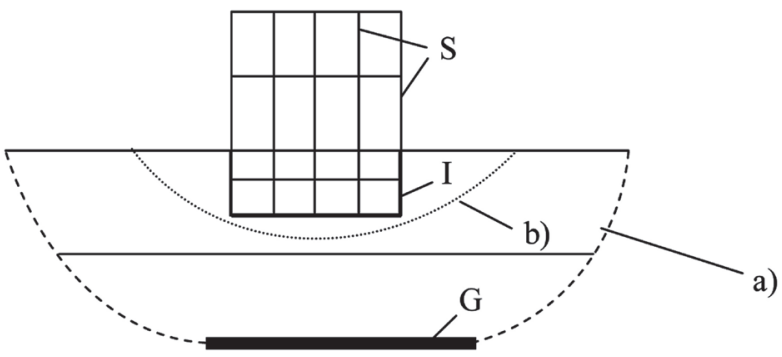

Fig. 2.1 Soil-structure system; boundary of model containing seismic source (a), boundary of model not containing seismic source (b).
$\tilde{\mathbf{K}}^{S}(\omega)=\sum_{e=1}^{E} \tilde{\mathbf{K}}^{e}(\omega)$.

In the case of a beam element the corresponding element relations are exact.

\subsubsection{Foundation}

In Fig. 2.1 we identify the soil-structure interface $\mathbf{I}$, and a (rigid) base rock $\mathbf{G}$, which is understood as the seismic source. We also use the concept of total displacements for the soil. The dynamic stiffness matrix of the soil (the foundation, sub-grade) has to be modeled through a procedure which allows waves to propagate to infinity. We mention here the Thin Layer / Flexible Volume Method, Lysmer, et.al. (1988), the Boundary Element Method,(Dominguez, 1993 ) or any other procedure based on Green's functions. These methods lead to a complex frequency dependent dynamic stiffness matrix of the foundation which we identify with the upper index $F$ (for the foundation)

$\tilde{\mathbf{K}}^{F}(\omega) \tilde{\mathbf{u}}^{F}(\omega)=\tilde{\mathbf{P}}^{F}(\omega)$

\subsubsection{System}

In the following for simplicity's sake we omit writing the tilde above the Fourier-transformed terms. Also, for the system's nodal variables we can omit the upper indices and denote with $\mathbf{u}_{I}$ the displacements on the structure-soil interface and with $\mathbf{u}_{S}$ the remaining structural degrees of freedom. For the sub-grade we assume that only the degree of freedoms, $\mathbf{u}_{I}$, on the interface and on the base rock, $\mathbf{u}_{G}$, are kept as primary unknowns. The two sub-structures are coupled using the direct stiffness method (e.g.,Bathe,1996) leading to the system equation

$$
\left[\begin{array}{ccc}
\mathbf{K}_{S S}^{S} & \mathbf{K}_{S I}^{S} & \mathbf{0} \\
\mathbf{K}_{I S}^{S} & \mathbf{K}_{I I}^{S}+\mathbf{K}_{I I}^{F} & \mathbf{K}_{I G}^{F} \\
\mathbf{0} & \mathbf{K}_{G I}^{F} & \mathbf{K}_{G G}^{F}
\end{array}\right]\left[\begin{array}{l}
\mathbf{u}_{S} \\
\mathbf{u}_{I} \\
\mathbf{u}_{G}
\end{array}\right]=\left[\begin{array}{l}
\mathbf{P}_{S} \\
\mathbf{P}_{I} \\
\mathbf{P}_{G}
\end{array}\right]
$$

\subsection{Excitation}

\subsubsection{Base rock excitation}

In the case of seismic base rock excitation we denote in equation (2.6) the prescribed motion with $\mathbf{u}_{G}=\overline{\mathbf{u}}_{G}$. Then $\mathbf{P}_{G}$ is the driving force corresponding to the prescribed base rock's motion. We further assume that the nodal forces $\mathbf{P}_{S}$ and $\mathbf{P}_{I}$ are zero and, not being interested in the driving force $\mathbf{P}_{G}$, for the structural unknowns $\mathbf{u}_{S}, \mathbf{u}_{I}$ write: 
$\left[\begin{array}{cc}\mathbf{K}_{S S}^{S} & \mathbf{K}_{S I}^{S} \\ \mathbf{K}_{I S}^{S} & \mathbf{K}_{I I}^{S}+\mathbf{K}_{I I}^{F}\end{array}\right]\left[\begin{array}{l}\mathbf{u}_{S} \\ \mathbf{u}_{I}\end{array}\right]=\left[\begin{array}{c}\mathbf{0} \\ \mathbf{P}_{e f f, I}\end{array}\right]$

In equation (2.7) the effective seismic force acting at the soilstructure interface $\mathbf{I}$ is

$\mathbf{P}_{e f f, I}=-\mathbf{K}_{I G}^{F} \overline{\mathbf{u}}_{G}$.

\subsubsection{Seismic field excitation}

If the seismic field is produced by a source outside the region analysed one can express the effective seismic forces through the seismic field without a structure. We distinguish two seismic fields:

a) the free field: seismic displacement field of the soil without any influence on the structure, Fig. 2.2 (a),

b) the scattered field: the displacement field without the structure but with an excavation, Fig. 2.2 (b).

\section{Scattered field excitation}

We assume first that the scattered field $\mathbf{u}^{\prime \prime}$ is known and that it may be deduced from the source's motion. We denote it as $\mathbf{u}_{I}^{\prime \prime}$ and $\mathbf{u}_{G}^{\prime \prime}=\overline{\mathbf{u}}_{G}$ at the interface and at the source, respectively. For purposes of clarity, we also introduce the upper index "for the dynamic stiffness and for the load for the site in Fig. 2.2 b). From equations (2.7) and (2.8) follows without the structure

$\mathbf{K}_{I I}^{F^{\prime \prime}} \mathbf{u}_{I}=-\mathbf{K}_{I G}^{F "} \overline{\mathbf{u}}_{G}$

If we define with

$\Delta \mathbf{u}_{I}=\mathbf{u}_{I}-\mathbf{u}_{I}^{\prime \prime}$

the difference between the actual displacement field and the scattered field, we obtain from equation (2.9)

$\mathbf{K}_{I I}^{F^{\prime \prime}} \Delta \mathbf{u}_{I}=\mathbf{K}_{I I}^{F^{\prime \prime}}\left(\mathbf{u}_{I}-\mathbf{u}_{I}^{\prime \prime}\right)=0$,

or

$\mathbf{K}_{I I}^{\prime \prime F} \mathbf{u}_{I}=\mathbf{K}_{I I}^{\prime \prime F} \mathbf{u}_{I}^{\prime \prime}=-\mathbf{K}_{I G}^{F \prime \prime} \overline{\mathbf{u}}_{G}=\mathbf{P}_{I}^{\prime \prime F}$.
Equation (2.12) is the definition of the scattered field in terms of the source motion.

If we insert the structure into the soil, the total displacement field will differ from the scattered field. On the structural side of the interface the created forces are

$\mathbf{P}_{I}^{S}=\mathbf{K}_{I S}^{S} \mathbf{u}_{S}+\mathbf{K}_{I I}^{S} \mathbf{u}_{I}$

These forces have to be in equilibrium with the interface forces on the soil side

$\mathbf{P}_{I}^{S}+\mathbf{P}_{I}^{\prime \prime F}=0$

With these conditions the equation of motion becomes

$\left[\begin{array}{cc}\mathbf{K}_{S S}^{S} & \mathbf{K}_{S I}^{S} \\ \mathbf{K}_{I S}^{S} & \mathbf{K}_{I I}^{S}+\mathbf{K}_{I I}^{\prime \prime F}\end{array}\right]\left[\begin{array}{c}\mathbf{u}_{S} \\ \mathbf{u}_{I}-\mathbf{u}_{I}^{\prime \prime}\end{array}\right]=\left[\begin{array}{l}\mathbf{0} \\ \mathbf{0}\end{array}\right]$

or

$\left[\begin{array}{cc}\mathbf{K}_{S S}^{S} & \mathbf{K}_{S I}^{S} \\ \mathbf{K}_{I S}^{S} & \mathbf{K}_{I I}^{S}+\mathbf{K}_{I I}^{\prime \prime F}\end{array}\right]\left[\begin{array}{l}\mathbf{u}_{S} \\ \mathbf{u}_{I}\end{array}\right]=\left[\begin{array}{c}\mathbf{0} \\ \mathbf{K}_{I I}^{\prime \prime F} \mathbf{u}_{I}^{\prime \prime}\end{array}\right]=\left[\begin{array}{c}\mathbf{0} \\ \mathbf{P}_{e f f, I}^{\prime \prime}\end{array}\right]$

\section{Free field excitation}

With the same arguments we obtain for a system consisting of the free field site and the structure

$\left[\begin{array}{cc}\mathbf{K}_{S S}^{S} & \mathbf{K}_{S I}^{S} \\ \mathbf{K}_{I S}^{S} & \mathbf{K}_{I I}^{S}+\mathbf{K}_{I I}^{F^{\prime}}\end{array}\right]\left[\begin{array}{l}\mathbf{u}_{S} \\ \mathbf{u}_{I}\end{array}\right]=\left[\begin{array}{c}\mathbf{0} \\ \mathbf{K}_{I I}^{F^{\prime} \mathbf{u}_{I}^{\prime}}\end{array}\right]=\left[\begin{array}{c}\mathbf{0} \\ \mathbf{P}_{e f f, I}^{\prime}\end{array}\right]$

with

$\mathbf{P}_{e f f, I}^{\prime}=\mathbf{K}_{I I}^{F^{\prime}} \mathbf{u}_{I}=-\mathbf{K}_{I G}^{F^{\prime}} \overline{\mathbf{u}}_{G}$

Equation (2.18) is the definition or the free field in terms of the source motion.

The difference between the free field site and the scattered field site is the excavation (see Fig. 2.3).
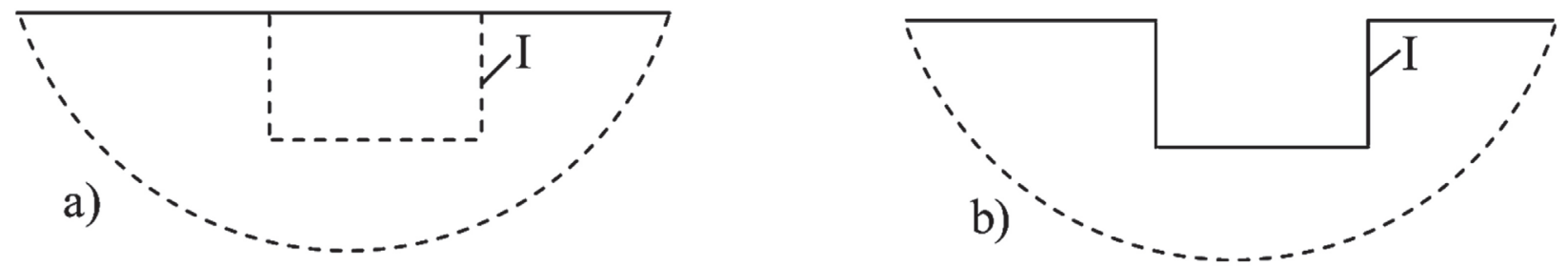

Fig. 2.2 Section of a sub-grade without a structure (a); section of a sub-grade with an excavation (b). 


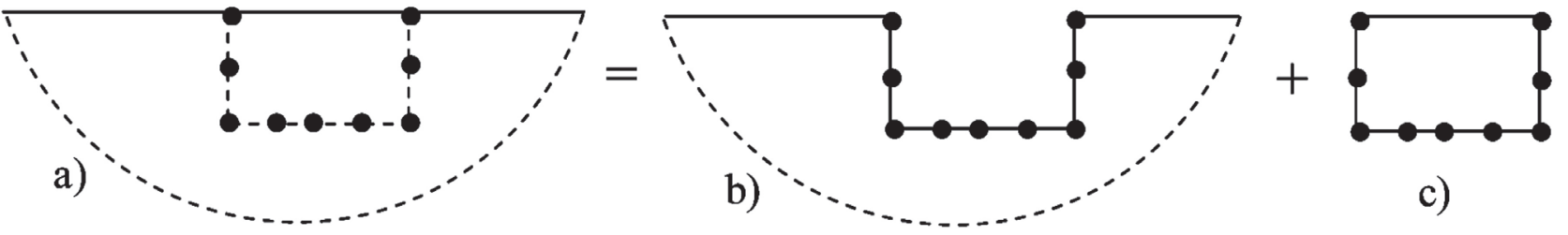

Fig. 2.3 Free field site (a); scattered field site (b); excavation (c).

The same is true for the corresponding dynamic stiffnesses

$\mathbf{K}_{I I}^{\prime F}=\mathbf{K}_{I I}^{\prime \prime F}+\mathbf{K}_{I I}^{E}$,

where $\mathbf{K}_{I I}^{\prime F}, \mathbf{K}_{I I}^{\prime \prime F}$ and $\mathbf{K}_{I I}^{E}$ are the dynamic stiffnesses of the free field site, the scattered field site and the excavation, respectively, condensed to the same degrees of freedom on the soil-structure interface. The corresponding nodal points are indicated in Fig. 2.3. Since in equation (2.17) the site is not yet excavated, we have to subtract the excavation $\mathbf{K}_{I I}^{E}$ from the free field impedance and obtain

$\left[\begin{array}{cc}\mathbf{K}_{S S}^{S} & \mathbf{K}_{S I}^{S} \\ \mathbf{K}_{I S}^{S} & \mathbf{K}_{I I}^{S}+\mathbf{K}_{I I}^{\prime F}-\mathbf{K}_{I I}^{E}\end{array}\right]\left[\begin{array}{l}\mathbf{u}_{S} \\ \mathbf{u}_{I}\end{array}\right]=\left[\begin{array}{c}\mathbf{0} \\ \mathbf{P}_{e f f, I}^{\prime}\end{array}\right]$

The free field excitation formulation can be generalized for additional "interaction points" which are not situated on the soilstructure interface. An example of such a procedure has been used by Lysmer, et al. (1988) in the SASSI computer program, where as interaction points, I, the points of the intersection between the soil and structure, have been chosen, as indicated in Fig. 2.4.

\subsection{Rigid Soil-Structure Iterface}

Due to a structural design or the introduction of simpler mechanical models, it is often assumed that a foundation, i.e., the soil-structure interface, is rigid. This results in a kinematic constraint for the
DOFs on the soil-structure interface (or possibly for the DOFs of the basement interaction nodes) since the motion can now be expressed through the rigid body DOFs of the interface. Let $\mathbf{u}_{0}$ be the rigid body DOFs related to a reference point $\mathbf{O}$, usually chosen as the center of the lower face of the embedment. Then we can establish the relation $\mathbf{u}_{I}=\mathbf{a} \mathbf{u}_{0}$ which leads, referring to equation (2.7), to the transformed stiffness relation (see e,g, Bathe, J., 1996)

$\left[\begin{array}{cc}\mathbf{K}_{S S}^{S} & \mathbf{K}_{S I}^{S} \\ \mathbf{a}^{T} \mathbf{K}_{I S}^{S} & \mathbf{a}^{T} \mathbf{K}_{I I}^{S} \mathbf{a}+\mathbf{K}_{0}^{F}\end{array}\right]\left[\begin{array}{l}\mathbf{u}_{S} \\ \mathbf{u}_{0}\end{array}\right]=\left[\begin{array}{c}\mathbf{0} \\ \mathbf{a}^{T} \mathbf{P}_{e f f, 0}\end{array}\right]$

In equation (2.21) $\mathbf{K}_{0}^{F}=\mathbf{a}^{T} \mathbf{K}_{I I}^{F} \mathbf{a}$ and $\mathbf{P}_{e f f, 0}=\mathbf{a}^{T} \mathbf{P}_{e f f, I}$ are the stiffness and effective load of the rigid foundation.

\section{RELATIVE DISPLACEMENT FORMULATION}

In classical structural dynamics where the mechanical modal is usually much simpler, the equation of motion is written in the case of base excitation in deformations relative to the rigid base of the structure. Then the effective earthquake forces are the inertia forces acting on the mass of the structure. This approach is also used in cases where the soil-structure interaction is considered and the excitation arises from a specified base rock motion or from a known free field motion. The advantage of this formulation lies in the fact that the seismic field motion and the rigid body motion

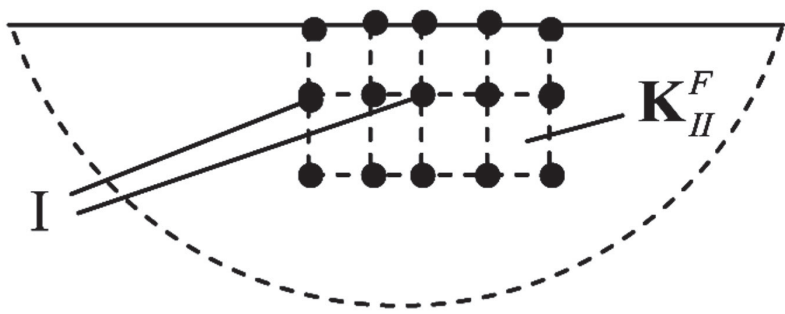

Fig. 2.4 Example of interaction points in Lysmer, et al. (1988).

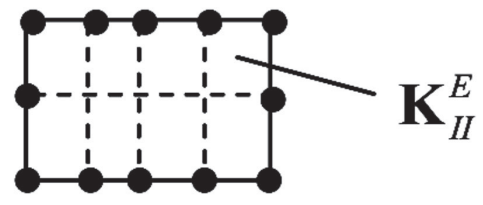


of the structure, i.e., the kinematic interaction, are eliminated from the unknowns; and also, that the effective earthquake forces are proportional to the acceleration of the seismic field, which are the variables measured in the strong motion records. But it should be noted that effective earthquake forces can only be expressed in inertial forces alone if no stresses are introduced through kinematic interaction into the structure. And this is generally only true if the base of the structure is rigid. In this case the kinematic interaction is equal to the rigid body motion of the structure and the stresses in the structure are only due to inertial forces.

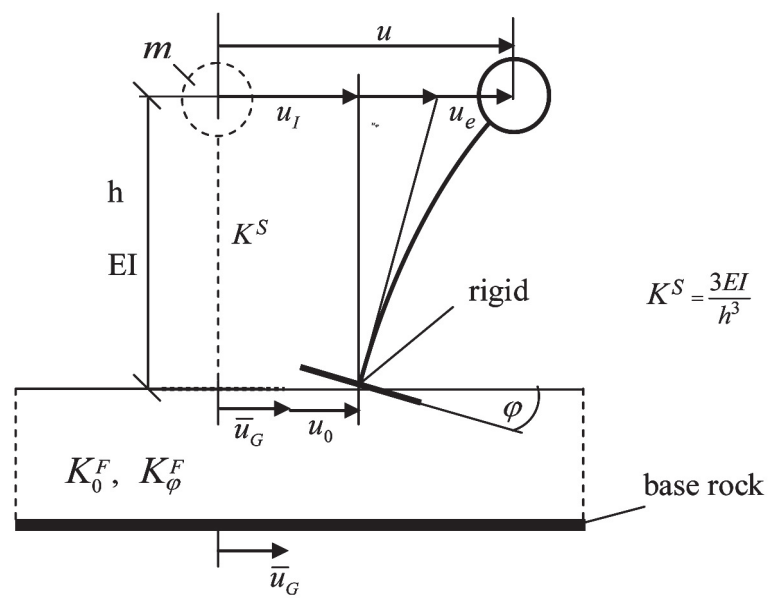

Fig. 3.1 Total and elastic displacements.

For the simple soil-structure model shown in Fig.3.1, with a bending stiffness $K^{S}$ resting on a soil layer with dynamic stiffnesses $K_{0}^{F}$ , $K_{\varphi}^{F}, K_{0 \varphi}^{F}=0$, and excited by a horizontal base rock motion $\bar{u}_{G}$ and horizontal free field motion $u_{I}^{\prime}$, respectively, we show the transformation from total to relative displacements. The direct stiffness method gives the equation of motion in total displacements

$$
\left[\begin{array}{cccc}
K^{S}-\omega^{2} m & -K^{S} & -h K^{S} & 0 \\
-K^{S} & K^{S}+K_{0}^{F} & h K^{S} & -K_{0}^{F} \\
-h K^{S} & h K^{S} & h^{2} K^{S}+K_{\varphi}^{F} & 0 \\
0 & -K_{0}^{F} & 0 & K_{0}^{F}
\end{array}\right]\left[\begin{array}{c}
u \\
u_{I} \\
\varphi \\
\bar{u}_{G}
\end{array}\right]=\left[\begin{array}{c}
0 \\
0 \\
0 \\
P_{G}
\end{array}\right] \text { or } \quad \mathbf{K u}=\mathbf{P}
$$

Base rock excitation

The relations between total displacements $u, u_{I}, u_{G}$ and the relative displacements $u_{e}, u_{0}, \varphi, u_{G}$ are

$u_{I}=u_{G}+u_{0} ; u=u_{I}+u_{\varphi}+u_{e}=u_{G}+u_{0}+h \varphi+u_{e}$ which in a matrix notation is $\left[\begin{array}{c}u \\ u_{I} \\ \varphi \\ u_{G}\end{array}\right]=\left[\begin{array}{llll}1 & 1 & h & 1 \\ 0 & 1 & 0 & 1 \\ 0 & 0 & 1 & 0 \\ 0 & 0 & 0 & 1\end{array}\right]\left[\begin{array}{c}u_{e} \\ u_{0} \\ \varphi \\ u_{G}\end{array}\right] \quad$ or $\quad \mathbf{u}=\mathbf{a u}_{e}$

The transformation of equation (3.1) with the transformation matrix a to the relative displacements $\mathbf{u}_{e}$ results with $\mathbf{K}_{e}=\mathbf{a}^{T} \mathbf{K a} ; \mathbf{P}_{e}=\mathbf{a}^{T} \mathbf{P}$ in

$$
\begin{aligned}
& {\left[\begin{array}{cccc}
K^{S}-\omega^{2} m & -\omega^{2} m & -\omega^{2} m h & -\omega^{2} m \\
-\omega^{2} m & K_{0}^{F}-\omega^{2} m & -\omega^{2} m h & -\omega^{2} m \\
-\omega^{2} m h & -\omega^{2} m h & K_{\varphi}^{F}-\omega^{2} m h^{2} & -\omega^{2} m h \\
-\omega^{2} m & -\omega^{2} m & -\omega^{2} m h & K_{0}^{F}-\omega^{2} m
\end{array}\right]\left[\begin{array}{c}
u_{e} \\
u_{0} \\
\varphi \\
\bar{u}_{G}
\end{array}\right]=\left[\begin{array}{c}
0 \\
0 \\
0 \\
P_{e}
\end{array}\right]} \\
& \text { or } \quad \mathbf{K}_{e} \mathbf{u}_{e}=\mathbf{P}_{e}
\end{aligned}
$$

or for the unknown structural displacements

$$
\left[\begin{array}{ccc}
K^{S}-\omega^{2} m & -\omega^{2} m & -\omega^{2} m h \\
-\omega^{2} m & K_{0}^{F}-\omega^{2} m & -\omega^{2} m h \\
-\omega^{2} m h & -\omega^{2} m h & K_{\varphi}^{F}-\omega^{2} m h^{2}
\end{array}\right]\left[\begin{array}{c}
u_{e} \\
u_{0} \\
\varphi
\end{array}\right]=\omega^{2} m \bar{u}_{G}\left[\begin{array}{l}
1 \\
1 \\
h
\end{array}\right]
$$

Free field excitation

The equation in total deformations is

$$
\left[\begin{array}{ccc}
K^{S}-\omega^{2} m & -K^{S} & -h K^{S} \\
-K^{S} & K^{S}+K_{0}^{F} & h K^{S} \\
-h K^{S} & h K^{S} & h^{2} K^{S}+K_{\varphi}^{F}
\end{array}\right]\left[\begin{array}{c}
u \\
u_{I} \\
\varphi
\end{array}\right]=\left[\begin{array}{c}
0 \\
K_{0}^{F} u_{I}^{\prime} \\
0
\end{array}\right]
$$

The transformation from total to relative displacement is written now as

$$
\left[\begin{array}{l}
u \\
u_{I} \\
\varphi
\end{array}\right]=\left[\begin{array}{lll|l}
1 & 1 & h & 1 \\
0 & 1 & 0 & 1 \\
0 & 0 & 1 & 0
\end{array}\right]\left[\begin{array}{c}
u_{e} \\
u_{0} \\
\frac{\varphi}{u_{I}^{\prime}}
\end{array}\right] \quad \text { or } \quad \mathbf{u}=\left[\begin{array}{lll}
\mathbf{a}_{e} & \mathbf{a}^{\prime}
\end{array}\right]\left[\begin{array}{c}
\mathbf{u}_{e} \\
\hdashline \mathbf{u}_{I}^{\prime}
\end{array}\right]
$$

The transformation of equation (3.5) with the transformation matrix (3.6) to the relative variables results with $\mathbf{K}_{e}=\mathbf{a}_{e}{ }^{T} \mathbf{K} \mathbf{a}_{e} ; \mathbf{P}_{e}=\mathbf{a}_{e}{ }^{T}$ $\left(\mathbf{P}-\mathbf{K a}^{\prime} \mathbf{u}_{I}{ }^{\prime}\right.$ in

$$
\left[\begin{array}{ccc}
K^{S}-\omega^{2} m & -\omega^{2} m & -\omega^{2} m h \\
-\omega^{2} m & K_{0}^{F}-\omega^{2} m & -\omega^{2} m h \\
-\omega^{2} m h & -\omega^{2} m h & K_{\varphi}^{F}-\omega^{2} m h^{2}
\end{array}\right]\left[\begin{array}{c}
u_{e} \\
u_{0} \\
\varphi
\end{array}\right]=\omega^{2} m u_{I}^{\prime}\left[\begin{array}{l}
1 \\
1 \\
h
\end{array}\right]
$$




\section{CONCLUSION}

Analysis of dynamic soil-structure interaction in a frequency domain together with the total displacement formulation is especially advantageous for structures with elastic embedded foundations: condensation to primary degrees of freedom is possible without a loss of accuracy; material damping for any rheological model can be considered easily; kinematic and inertial interaction are obtained together utilizing the usual FEM-procedure; and even nonlinear problems may be investigated. For the simple case of a beam with a rigid surface foundation [3] the total displacement formulation is compared with the classical approach where the unknowns are the elastic deformations relative to the rigid base of the structure. Numerical examples, showing the obtained resuts, were published in [8].

\section{REFERENCES}

[1] Bathe, J. (1996). Finite element procedures. Prentis-Hall, Englewood Cliffs, N.J., USA

[2] Hillmer, P. and Schmid, G. (1988). Calculation of foundation uplift effects using a numerical Laplace transform. Earthquake Engineering and Structural Dynamics, 16, 789-801

[3] Kolekova, Y. and Schmid, G. (2004). Remarks on dynamic soilstructure interaction. Proceedings of the JDGK-Symposium o4, 2, 235-241.

[4] Lysmer, et al (1988). SASSI: a system for analysis of soilstructure interaction. University of Berkeley

[5] Matthees, W. (1982). A strategy for the solution of soil dynamic problems involving plasticity by transform. Journal for Num. Meth. in Engineering 18, 1601-1611.

[6] Schmid, G. and Chouw, N. (1992). Soil-structure interaction effects on structural pounding, Earthquake Engineering. Tenth World Conference, Balkema, Rotterdam, 1651-165

[7] Wolf, J.P. (1985). Dynamic soil structure interaction. PrenticeHall, Englewood Cliffs, N.J., USA

[8] Koleková, Y., Petronijević, M., Schmid, G.: "Special dynamic soil-structure analysis procedures demonstrated for two towerlike structures."Slovak Journal of Civil Engineering, volume XVIII, 2010/2, pp.26-33. 